\title{
CONTEMPLACIÓN DE LO NATURAL Y ACCIÓN HUMANA EN LA MAGIA: SAN AGUSTIN Y PICO DELLA MIRANDOLA
}

Silvia Magnavacca*

SINTESIS - Este trabajo se propone mostrar que la concepción de magia de Pico della Mirandola, de un lado, es la propia de un humanista; de otro, responde más de lo que se suele creer a líneas tradicionales. Para ello, y después de determinar la significación precisa de algunos términos a emplear, en primer lugar, examina aspectos de la posición agustiniana sobre el tema. En segundo término, estudia el tratamiento de las tesis piquianas en tres etapas, constituidas por la Oratio con las Conclusiones, la Apologia, y las Disputationes. En tercer lugar, refuta la interpretación que ve en estas últimas una retractación de Pico respecto de sus primeras posiciones sobre la magia. Por último, muestra que las divergencias entre ambos autores obedecen, sobre todo, a una cuestión terminológica y de matices, destacando, no obstante la relevancia de éstos.

PALABRAS-CLAVE - Augustin. Picco della Mirandola. Magia. Contemplación. Acción humana.
ABSTRACT - This work intends to show that the conception of magic of Pico della Mirandola, of a side, it is the characteristic of a humanist; of other, it responds more than what is usually believed to traditional lines. For it, and after determining the precise significance of some terms to use, in the first place, it examines aspects of the augustinian position on the topic. In second term, it studies the treatment of the Pico della Mirandola's thesis in three stages, constituted by the Oratio with the Conclusions, the Apologia, and the Disputationes. In third place, it refutes the interpretation that sees in these last ones a retraction of Pick regarding their first positions on the magic. Lastly, it shows that the divergences between both authors obey, mainly, to a terminological question and of shades, highlighting, nevertheless the relevance of these.

KEY WORDS - Augustin. Pico della Mirandola. Magic. Contemplation. Human action.

Entre los equívocos frecuentes en la estimación del Humanismo renacentista tiene un peso no desdeñable - como suele suceder en la historia de la filosofía - la descontextualización de su terminología. Esto también ocurre con la noción de magia entre los humanistas. Dejaremos a un lado, en pro de la precisión, otros vocablos conexos, como "alquimia", cuya importancia y frecuencia son más tardías. Cargada de un sentido negativo, que le fuera otorgado en el período patrísti-

* Universidad de Buenos Aires. Consejo de Investigaciones Científicas y Tecnológicas - CONICET, Buenos Aires.

\begin{tabular}{|l|l|l|l|l|l|}
\hline VERITAS & Porto Alegre & v. 44 & n. 3 & Setembro 1999 & p. 831-842 \\
\hline
\end{tabular}


co y durante un largo trecho de la Edad Media, la palabra "magia" puede leerse prejuiciadamente en las obras humanísticas, en las que, en general, se vuelve más a los textos patrísticos que a los tardo-medievales.

En el caso particular de San Agustín, que examinaremos brevemente para detenernos después en el piquiano, el término "magia" aparece siempre con connotaciones clara y rotundamente negativas ya desde su misma caracterización. En efecto, Agustín la indica como "arte de impía curiosidad" (nefariae curiositatis ars) en De civ. Dei X, 9, 1, insistiendo a lo largo de todo este capitulo en su condición demoníaca, ya anunciada en el inmediato anterior. Cabe señalar, con todo, que establece en esas líneas un interesante matiz de diferencia entre "magia" y goeteia, desafortunadamente no explicitado: al referirse a los hechizos compuestos por el arte de impia curiosidad, dice literalmente que se designan con el nombre de magia "o con el nombre más detestable de goeteia".

Con especial encarnizamiento polémico, el Hiponense ataca la magia egipcia en XVIII, 39 de la misma obra, así como en la Epist. 139, 13 y en el Sermón de la Montaña, SM I, 25, 85. Un tono aún más severo revisten el pasaje de la Epist. 138, 19 , en el que, refiriéndose a Apuleyo y sus defensores, habla del "crimen de las artes mágicas", y el de In Io. Ev. 97, 3 que alude a los "ritos nefandos" de estas artes.

Con todo, hay dos textos agustinianos al respecto que merecen particular atención, dado que en ellos se apunta a una distinción de gran importancia para nuestro tema. Es un hecho que la magia y todo lo que le concierne presenta la nota de revelación de lo oculto para la mayoria y, por ende, de lo espectacular, como el milagro y la profecía; de ahí la preocupación del obispo de Hipona por separar las aguas ante los ojos de los más, quienes constituian su grey. Así, mientras en la Epist. 138, 20, advierte sobre la no equiparación de los magos con los profetas, en De civ. Dei XXI, 6 marca la diferencia entre la actividad del mago, entendido como brujo, y lo que hoy llamariamos una operación técnica sobre el ámbito de lo natural, en una explicación cuyo interés merece un examen literal. Es ésta: los demonios, artífices de las artes mágicas, se procuran, sobre todo, la amistad de los hombres, conociendo el corazón de cada uno y seduciéndolo. Estos demonios "son atraídos para morar en ciertos lugares, por ciertos signos apropiados a su gusto, como diversas clases de piedras [...] de encantamientos y ceremonias". Agustín insiste en que "se afanan principalmente por poseer los corazones de los mortales, y esta posesión es su principal gloria". En cambio,

"[...] las creaturas de Dios obran por medio de artes esas maravillas que llaman 'mechanémata' tan sorprendentes, que quienes no las conocen las creen divinas. Así vemos que una estatua de hierro suspendida, en algún cuarto, por dos piedras magnéticas de igual tamaño y peso, colocadas una en el techo y otra en el suelo, se mantiene en el aire. Quienes ignoran la razón de esto, lo llaman 'milagro' [...] Esas maravillas, increíbles para los infieles, son fáciles y hacederas para Dios, para ese Dios quien ha dado tal virtud a las piedras y a los demás seres, y quien ha dado el ingenio a los hombres, los que las utilizan de mil modos admirables [...]."

De civ. Dei XXI, 6, 2. Esta traducción es propia, como todas las que siguen, salvo expresa indicación en contrario. En este caso, nos hemos basado, retocándola, sobre la ofrecida por Morán, en la edición de Madrid, BAC, 1965, p. 628. Subrayado nuestro. 
Nótese, en primer lugar, que Agustín juzga como típica intención de los demonios el valerse del estupor del hombre para conquistar en él confianza en el poder demoníaco. En segundo término; ese estupor proviene de la ignorancia de fenómenos naturales, tal como lo indica el ejemplo aducido de las piedras magnéticas. En tercer lugar, se distingue entre el plano natural y el sobrenatural, sea este último divino, angélico o demoníaco. Por último, y esto es definitivo, las "maravillas" propias de los fenómenos naturales, se atribuyen a la economía misma del Ordo universalis, cuyo autor es Dios mismo, como todo el capítulo siguiente se dedica a subrayar.

Ya en una obra patrística como ésta aparecen notas que son comunes a todo discurso sobre la magia y que resurgen, como veremos, en el piquiano. La primera de ellas es lo que Kieckhefer ha llamado "los sentidos subjetivo y objetivo de lo oculto". En efecto, al preguntarse por el significado de los poderes ocultos en la naturaleza, tan íntimamente ligados con la noción de magia, este autor señala que, muchas veces, los textos parecen aludir con ello a "lo que está más allá de los sentidos, de manera que la mayoría de la gente no puede captar cómo ocurren o a qué causas obedecen". Se remite aquí a una célebre afirmación de Picatrix. ${ }^{3}$ Pero observa que, más allá de esto, la palabra "magia" también ha tenido un sentido más técnico, por asi decir, referido no a respuestas subjetivas sino al status objetivo del poder en cuestión.

En este último plano, el objetivo, es posible hablar, a su vez, de varios sentidos de lo oculto en los fenómenos. Según el primero, que podríamos denominar "intrínseco y específico", muchas propiedades de piedras, hierbas o animales se pueden explicar en términos de su estructura física interna, la cual obedece a la especie a la que cada ser pertenece. Así, para remitirse a ejemplos aristotélicos, una planta considerada húmeda o fría - características que debe a la especie de la que ella forma parte - se aconseja para el tratamiento de una enfermedad cuya causa se atribuye al exceso de calor o sequedad.

Ahora bien, hay otros casos, que por nuestra parte denominaríamos "extrínsecos y universales", en los cuales se afirma que el poder de modificación, curación o prevención proviene de algo externo a la estructura del objeto, por ejemplo, influencias de astros y planetas. En tales casos, se tiene en cuenta el orden universal considerado en su totalidad pero, además, en su condición natural. En otros términos, se tiene en cuenta la Naturaleza en su conjunto y las profundas - y secretas - relaciones que en su seno están establecidas de hecho. En este contexto, poco importa la distancia o la consustancialidad entre dos entes para suponer que entre ambos puede darse una cierta relación que el mago está llamado a actualizar.

Por extensión, se ha asumido también, a través de los siglos, un tercer sentido "objetivo" de lo oculto en la magia: es el que se supone "fundado" en algún rasgo más que en la estructura interna del objeto que se cree dotado de cierto

Cfr. Kieckhefer, R., Magic in the Middle Ages, Cambridge, Cambridge Univ. Press, 1993, p. 12 y ss.

Cfr. Picatrix. The Latin Version of the Ghayat Al-Hakim, ed. David Pingree, London, Warburg Institute, 1986,5 . 
poder. Así, por ejemplo, se creyó que plantas cuyas hojas tienen forma de moneda pueden procurar riqueza a quien las posee o cultiva. Esta relación puramente simbólica de semejanza se asume, entonces, como relación de causa a efecto; de ahí que prefiramos llamar aquí "simbólico" a este sentido de lo mágico, aunque Frazer lo haya denominado "simpatético", para no generar equívocos con lo que, según veremos, en este contexto, es la sympátheia a la que Pico se refiere.

Sea de todo esto lo que fuere, lo cierto es que, en lo que concierne a la discusión piquiana sobre la magia, es fundamental tener presente, especialmente, el segundo sentido "objetivo" de los indicados, es decir, el "extrínseco y universal". Vayamos, pues, a dicha discusión.

Está ya claro entre los estudiosos del Mirandolano que sus posiciones sobre el tema que nos ocupa se disciernen en tres etapas, independientemente de la valoración hermenéutica que se quiera conceder a cada una de ellas o a su división misma. Esas etapas están constituidas por la Oratio de dignitate hominis seguida por las Conclusiones o Tesis, la Apologia, y las Disputationes adversus Astrologiam Divinatricem. Tampoco está en discusión la intención desmistificadora de la magia que subsiste en Pico a lo largo de esos tres momentos, aunque los intérpretes no concuerdan a la hora de ponderar esa intención, como tampoco coinciden en la orientación de la evolución piquiana al respecto.

Tal intención se advierte ya en la primera etapa, en las páginas del Discurso sobre la dignidad del hombre, que Pico consagra precisamente a la diferenciación entre magia natural y goeteia. Así, dice:

"[...] la magia es doble: una se funda exclusivamente en la obra y autoridad de los demonios, cosa completamente execrable y monstruosa; la otra, bien mirada, no es sino el cabal cumplimiento [consummatio] de la filosofia natural. Los griegos tuvieron ambas en cuenta, y llamaron a una 'brujeria' [goeteian], para no dignarla con el nombre justo y especifico de 'magia' [mageian], a la que llaman perfecta y suprema sabiduría. De hecho, como dice Porfirio, en lengua persa, 'mago' significa 'el que es intérprete y cultor de las cosas divinas'. Grande, pues, muy grande es la disparidad entre estas artes. Una es condenada y execrada no sólo por la religión cristiana, sino por todas las leyes, por cualquier estado bien ordenado; la otra, la aprueban y abrazan los sabios [...]." ${ }^{5}$

Así, contrapone magia y goeteia no sólo en su denominación, es decir como antónimos, sino que, al par que deplora la segunda, reivindica la legitimidad de la primera a través de tres instancias: la filosófico-científica, la religiosa y aun la jurídica.

Cabe añadir que la goeteia es también llamada algunas veces "magia espiritual" en alusión no sólo a los espíritus perversos que en ella intervienen, sino también con el objeto de subrayar el carácter natural de su opuesta. Para acentuar, si

Cfr. Frazer, J.G., The Golden Bough, London, Macmillan, 1913, p. 54 y ss.

De hominis dignitate, Heptaplus, De ente et uno e scritti vari (a cura di E. Garin), Firenze, Vallecchi, 1942, p. 148. La cita de Porfirio corresponde a De abstinentia IV, 16. 
cabe, esa antítesis que reclama la legitimidad de las artes mágicas naturales, convoca en su auxilio a la tradición antigua más respetada en su círculo: la neoplatónica, como lo prueba no sólo la mención de Porfirio sino también la de Plotino que se lee unas líneas más abajo.

Aun el joven impetuoso y ciertamente incauto que Pico era en el momento de redactar la Oratio debe haber tenido en cuenta, por una parte, la más que probable resistencia que la inclusión de tales artes en la "cultura oficial" había de despertar; por otra, su énfasis hace sospechar que esa distinción, aunque conocida por los más cultos, podía no estar presente en la mayor parte de los miembros de su eventual auditorio, como sabemos que efectivamente ocurrió. Recuérdese que la Oratio de dignitate hominis fue redactada como discurso de apertura al debate sobre sus 900 tesis que el Mirandolano proponía llevar a cabo en Roma. La discusión se prohibe objetando algunas de dichas tesis, entre las que figuran las relativas a la magia.

Antes de acercarnos a éstas, para examinar otras aristas de la posición piquiana al respecto, detengámonos en aspectos importantes fijados en el mismo Discurso. Los dos más importantes conciemen a notas esenciales del ámbito en el que opera la magia natural y al fundamento mismo de su obrar. Respecto de lo primero, dice:

"[...] abraza la contemplación más alta de las cosas más secretas y el conocimiento de toda la naturaleza. Desde el sancta sanctorum de ésta trae a la luz las fuerzas dispersas y diseminadas en el mundo por la bondad de Dios y, más que hacer milagros, sirve fielmente a la milagrosa naturaleza. Ella escruta en profundidad el consenso del universo, que expresivamente los griegos llaman sympátheia, explora la recíproca relación de las naturalezas [...]. ${ }^{n 6}$

El conocimiento de la magia, ya descrita como culminación del saber referido a las cosas de este mundo, concierne, pues, a la Naturaleza en su conjunto, esto es, a lo que Agustín llamaba el Ordo universalis. Son sus virtudes seminales - y, por ende, ocultas a la simple mirada de los hombres - aquello que el mago descubre y con lo que trabaja. Pero, además, el pasaje contiene dos observaciones de gran importancia: en primer lugar, subraya - casi se diría que en tono agustiniano - que la magia no hace milagros; muestra los llamados tales, en el sentido de maravillas, que subyacen en el mundo natural. Con ello, Pico mantiene estrictamente en el orden inmanente la actividad mágica, con explicita exclusión de cualquier intervención sobrenatural.

En segundo término, esas maravillas lo son, sobre todo, por las invisibles relaciones de consenso que unen a los seres entre sí: aunque éstos estén separados por la distancia física, es decir diseminados, hay entre ellos relaciones de sintonia, sea por semejanza o aun por oposición, dadas por su estructura, pero no tomada en sí misma, sino en la relación que ella guarda con la estructura del universo como tal. Es esa trama de relaciones entre las especies lo que Pico llama aquí "simpatía" cósmica. Así pues, la piquiana es una concepción "objetiva" de lo

6 Ibid. p. 152. La alusión a los griegos está registrada por Plinịo en Nat. Hist. XX, 1. Subrayado nuestro. 
oculto, puesto que las maravillas se atribuyen al mundo natural y no al poder sobrenatural o espiritual del mago, y responde, además, al tipo que hemos llamado "extrínseco y universal".

El texto avanza ahora en la dirección del obrar del mago sobre esas bases:

"[...] y, como el campesino casa los olmos con las vides, así el mago casa la tierra con el cielo, o sea, las fuerzas inferiores a las dotes y propiedades supremas. Por eso, lla goeteia, la brujerial, haciendo al hombre siervo de los enemigos de Dios, lo aleja de Él, mientras que la magia lo eleva a la admiración de las obras del Señor [...]."

De esta manera, los poderes del mago son, por cierto, excepcionales. Pero lo son precisamente en virtud de su conocimiento y manejo de medios naturales. Respecto del primero, hay de prestar atención al hecho de que tal conocimiento no constituye una revelación, sino un saber más profundo de la economía del mundo natural. En relación con el segundo, se ha de decir que ese manejo no es una manipulación, sino una actualización de las potencias naturales, que se lleva a cabo fundamentalmente por la unión de las que el mago sabe "consonantes".

Decíamos que esta primera etapa o momento está conformada no sólo por la Oratio sino también por las 900 Conclusiones o Tesis que le siguen. De ellas, Pico dedica 26 al tema que nos ocupa, en las cuales no hace sino confirmar estas mismas concepciones, expresándolas en la forma nítida y escueta que conviene a la enunciación de una tesis. Así, por ejemplo, en las Conclusiones 3 y 4 se lee que "la magia es la parte práctica de la ciencia natural" y que "por ello, constituye el aspecto más noble" de ésta. ${ }^{7}$ Conviene notar un matiz significativo en estas tesis: su tonalidad renacentista.

En efecto, el beneficio de la magia no radica en el aspecto cognoscitivo, meramente especulativo, aun cuando, como señalaba el Discurso, éste puede conducir a la admiración por la grandeza de Dios a través de sus obras; su carácter benéfico estriba en el servicio concreto que ella presta al hombre, quien, durante el Humanismo recupera el centro de la escena.

En las Tesis 11 y 13 se reiteran los fundamentos del obrar mágico. En ellas se dice que "los prodigios del arte mágica no tienen lugar sino por la unión y actualización de esas fuerzas que están en la naturaleza seminaliter et separata" y que "hacer operaciones mágicas no es otra cosa que enlazar al mundo". Pero es en la Tesis 5 donde se encuentra, quizá, la más optimista y escandalizadora de las afirmaciones piquianas al respecto, puesto es la que concede a ese obrar el mayor alcance. Dice, en efecto, que "no hay en el cielo y en la tierra, seminaliter et separate, ninguna virtud que el mago no pueda unir y llevar al acto". Esto ha impulsado a Paola Zambelli a hablar de utopía en la concepción de magia propia de Pico. ${ }^{8}$ Aun sin acordar con cierta nota de exageración que encontramos en sus afirmaciones, lo cierto es que, una vez subrayado el arduo conocimiento de lo natural que se exige al mago, Pico extrema los alcances de su acción.

7 "Magia est pars practica scientiae naturalis" y "Ex ista conclusione [... sequitur, quod magia sit nobilissima pars scientiae naturalis". Se sigue la edición de Conclusiones sive Thesis DCCCC, introducción y notas críticas de B. K̇ieszkowski, Genève, Droz, 1973.

Cfr. Zambelli, P., L'ambigua natura della magia. Filosofi, streghe, niti nel Rinascimento, Milano, Il Saggiatore, 1989, p. 182. 
No obstante, no creemos que ésta llegue a involucrar en el Mirandolano el carácter objetivo de lo oculto que hemos llamado "simbólico" ut sic. Frances Yates sugiere que el uso de talismanes se debería incluir entre los métodos mediante los cuales el mago natural piquiano "une" potencias celestiales con las terrenas, o casa el mundo, lo cual, para esta autora, significa lo mismo. ${ }^{3}$ El significado de ambas expresiones es en realidad el mismo, sólo que, en nuestra opinión, lo es en el sentido de que las potencias son celestiales en cuanto rasgos de las formas o especies contenidas en el Logos divino, es decir, en el diseño arquitectónico del universo. Nos parece que en esta dirección se ha de pensar el ejemplo del olmo y la vid aportado por el mismo autor en la Oratio. Por otra parte, Thorndike aporta interesantes elementos históricos que quitan peso a la suposición de Yates al respecto. ${ }^{10}$

Cabe ponderar también un testimonio relevante, no ya de su sobrino Gian Francesco -respecto del que es válida cualquier reserva, dada su condición apologética- sino de Benivieni. En una carta redactada durante la primera estancia del Mirandolano en Florencia, aquél da cuenta de que la preocupación de Pico tanto por la magia como por la Cábala no obedecía al interés de hacer conjuros, milagros o profecías, sino que era puramente erudita y se fundaba en su intención de enriquecer con esos elementos un comentario sobre el conjunto de las Escrituras" que tenía el propósito de escribir, cosa que llevó a cabo años después con el Heptaplus. Lo significativo de este documento de Benivieni radica en lo que permite confirmar y suponer: de un lado, indica claramente que Pico no se proponia dedicarse, personalmente, a la actividad mágica en cuanto tal; en tal sentido, su opción siempre ha sido por la vida contemplativa. De este modo, sus disquisiciones sobre la magia natural permanecen en el plano teórico; más aún, el valor de aquéllas consiste en que dan cuenta de su teorización sobre el mundo natural. De otro lado, la época en que esta carta fue redactada deja pensar que aún antes del combate por la disputa romana sobre las 900 Tesis, las prejuiciadas sospechas sobre la personalidad intelectual de Pico ya se habían cemido sobre él, al menos, lo bastante como para que su círculo de amigos intentara salirles al quite.

Como se sabe, la tan temida reacción de la cultura oficial y, sobre todo, de los círculos eclesiásticos, tuvo lugar a fines del 1486. En un gesto que anticipa históricamente el de Lutero, el Mirandolano imprime y fija, en universidades europeas y en los ginnasi italianos, estas Conclusiones nongentae in omni genere scientiarum. ${ }^{12}$ Las funestas repercusiones culminan en la ya conocida frustración del proyecto y en órdenes papales de arresto contra él, condenando las Tesis como

9 Yates, F., "Giovanni Pico della Mirandola and Magic", en L'opera e il pensiero di Giovanni Pico della Mirandola nella Storia dell'Umanesimo, Firenze, Ist. Naz. di Studi sul Rinascimento, 1965, vol. I, p. 164.

Thomdike consigna una carta de Baptista de Mantua en la que éste relata un sueño habido poco después de la muerte de Pico. En él, el Mirandolano condenaba el uso de talismanes como supersticioso. Aun cuando se trata de una imagen onírica, es verosímil que ella reproduzca la impresión que el círculo que rodeaba a Pico tenía de sus opiniones. Cfr. A History of Magic and Experimental Science, New York, Columbia Univ. Press, 1954, vl IV, p. 511.

11 Cfr. citado por R. Marcel en su Marsile Ficin, Paris, 1958, p. 34.

12 Se cree que la irónica acotación "de omni re scibili" reproduce un comentario con que Ficino aludió a la convocatoria piquiana al debate. En cambio, se ha atribuido a Voltaire el añadido hiperbólico, siglos después, del "et quibusdam aliis". 
"escandalosas y sospechosas de herejía". Fue el tono, aunque no el contenido, heterodoxo de algunas de ellas lo que dio pie a esta reacción. Pero, sobre todo, fue la posición central de Pico: la búsqueda de una pax philosophica que sirviera de base doctrinal al ecumenismo y que se sustentaba en la convicción de un saber absoluto. Éste, si bien se encontraba en la superficie fraccionado y disperso, subyacía, reconocible, en los distintos sistemas filosóficos y en el bagaje sapiencial de las más diversas procedencias. De él forma parte la magia natural. Pero insistamos en que las tesis que le conciernen son más escandalizadoras que heterodoxas. De las trece que finalmente no logran pasar el examen eclesiástico, sólo una toca el tema que nos ocupa y sobre ella volveremos: es la que sostiene que la Cábala y ciertas modalidades de la magia son lo que mejor certifica la divinidad de Cristo, puesto que probarían que sus milagros fueron tales y no obras de manipulación de la naturaleza.

Sea de ello lo que fuere, la condena provoca la consternación de Pico, quien, en veinte noches redacta su Apologia. Ciertamente no publicada, ésta circuló, con todo, entre sus amigos en forma manuscrita, acompañando una reedición de reducido número de las Conclusiones.

Lo referido a las artes mágicas en la Apologia no hace sino ratificar los puntos de vista ya explicitados en las Tesis al respecto. Pero se advierte la insistencia en algunos aspectos de la cuestión que pudieron haber suscitado, al menos, resistencia o perplejidad. En este sentido, son particularmente importantes los siguientes puntos. En primer lugar, el Mirandolano subraya el carácter arduo de esta culminación del saber sobre lo natural, puesto que le interesa imputar la condición inquietante de lo oculto a lo que hemos denominado "el sentido subjetivo". Así, señala que, si la práctica de la magia es difícil, ello obedece precisamente al hecho de que presupone un exacto y perfecto conocimiento de todas las cosas naturales". ${ }^{13}$ Con ello, insiste, una vez más, en la compleja economía del mundo natural en el que ella se da. En segundo término, explicita lo anterior señalando que los poderes del mago, sin duda, son excepcionales, lo cual no significa que sean sobrenaturales: la magia no enseña nada más que a llevar a cabo obras que generan estupor por su nota insólita, al manejar poderes naturales y aplicarlos a sus pacientes igualmente naturales. ${ }^{14}$ Un poco más adelante vuelve aún sobre la intención desmitificadora, al decir, literalmente, "el mago opera sólo actualizando o uniendo las virtudes naturales". ${ }^{15}$

No es lo mismo promover algunas de estas virtudes o potencias, al modo como se aúnan el olmo y la vid, que convertir el agua en vino: el conocimiento exhaustivo de las leyes más profundas del mundo natural implica el reconocimiento de sus límites. De ahí que sólo el mago, es decir, quien tiene cabal conciencia de los mismos, pueda certificar que las maravillas espectaculares obradas por Cristo son efectivamente milagros, en la medida en que no responden en ese caso a la unión de potencias ni a la actualización de ellas, sino a una irrupción del orden de lo sobrenatural.

13 Cfr. Apologia Ioannis Pici Mirandulani Concordiae Comitis, ed. Francesco del Tuppo, Napoli, 1487, f. 50.

${ }_{14}$ Cfr. ibid., f. 49.

15 "[...] per istam magicam nihil operamur nisi solum actuando et uniendo virtutes naturales", ibid., f. 52. 
Quebrantado el sueño de la pax philosophica, el Mirandolano cejará en su empeño de conciliación y, con ello, de desmistificación de lo que se creía diabólico y él juzgaba valioso. Bajo la influencia de Savonarola - menor, empero, de lo que su sobrino Gian Francesco ha querido mostrar - se inclinará a la redacción de obras de carácter religioso, como el mismo Heptaplus y el comentario al Pater. Por eso, en la última etapa de su vida, se limitará a fundamentar posiciones del Cristianismo.

Después de su fracaso en el intento de hallar apoyo para la causa ecuménica en el seno de la Cristiandad, y, sobre todo, del clero, después de haber encontrado en él a enemigos más que a aliados - justamente por incomprensión - el ímpetu juvenil que lo había lanzado a la lucha está definitivamente quebrado. Entonces, libra solamente aquellos combates en los que sabe que "los suyos" no podrán sino estar de su lado, no tendrán nada que objetar. Cede, pues, a las instancias de Savonarola, quien lo impulsaba a redactar una obra contra las supercherías astrológicas: las Disputationes adversus astrologiam divinatricem.

En nuestra opinión, se ha dado un gran equívoco en el enfoque interpretativo que, en general, se confiere a esta obra. Tal equívoco deriva de una visión más amplia acerca de la evolución del itinerario de Pico: él renuncia a la defensa pública de sus ideas, no a ellas. Pero, tanto su acercamiento personal de los últimos años a la figura de Savonarola, como el diseño, ciertamente interesado, de su biografía que un fanático savonaroliano como Gian Francesco Pico hace en la Vita de Giovanni, han llevado a desenfocar la lectura de las Disputationes. Centradas en el problema astrológico, en ellas, el Mirandolano sólo roza el de la magia. Con todo, las alinea en la misma dirección.

Ahora bien, lo fundamental es que bajo el improbable supuesto de una "conversión" a la ortodoxia, se descontextualizan nociones y aun términos. En este sentido, es sorprendente que, en nuestros días, una antigua sugerencia de Lynn Thorndike haya sido seguida sin revisiones puntuales por Paola Zambelli y, especialmente, por Louis Valcke. ${ }^{18}$ No obstante la prolijidad piquiana de haber titulado su texto contra la astrología con la acotación divinatricem, esto es, iudiciaria, para diferenciarla de la astrologia tout court - o sea, de lo que hoy llamaríamos "astronomia" - se interpreta su contenido como si en éste atacara cualquier influencia de los astros, planetas y satélites sobre el resto de la naturaleza. Pero desacreditar la validez de los horóscopos, por ejemplo, no implica poner en tela de juicio nuestros actuales conocimientos sobre la influencia de la luna en las mareas. Lo notable en la persistencia de esta interpretación, que juzgamos errada, es que ni siqui-

16

Cfr. Thomdike, L., op. cit., p. 485, donde resuelve tratar las menciones de Pico en dos capítulos distintos para "separate his earlier attitude and his later attack on astrology"; por su parte, Paola Zambelli, en op. cit., p. 183, sostiene que con las Disputationes "egli è divenuto il massimo rappresentante di una posizione contraria a questa della sua giovinezza". Louis Valcke es aún más taxativo en su "Magic et miracle chez Jean Pic de la Mirandole", en Atti del Convegno su Giovanni Pico della Mirandola nel V Centennario della morte, Firenze, Ist. Naz. di Studi sul Rinascimento, 1996, vol. II, p. 164-166, donde habla de un "reversement de perspective" en la posición piquiana sobre la magia. 
era tiene en cuenta el taxativo pasaje del Prefacio del 1491, donde Pico advierte advertencia válida para todo el resto de la obra, puesto que de un prefacio se trata - sobre el equívoco posible:

"Cuando digo 'astrología', no entiendo la que mide el tamaño y los movimientos de las estrellas con método matemático, disciplina segura y noble, llena de dignidad por sus méritos y ampliamente sostenida por la autoridad de hombres doctisimos, sino aquella que prevé el futuro a partir del curso de los astros, especulación mentirosa, prohibida por las leyes religiosas y civicas, defendida por los charlatanes, sospechosa para todos los justos y todos los sabios." ${ }^{17}$

Así como la superchería de la astrologia divinatrix queda alineada con la propia de la goeteia, la validez de la astrología entendida como astronomía, en su sentido contemporáneo, queda alineada con la magia natural. En tal sentido, es Henri de Lubac quien vio claramente la cuestión, al sostener que nuestro autor ha reivindicado los derechos de la verdadera astrología, así como los de la verdadera Cábala y los de la verdadera magia. ${ }^{18}$ Por cierto, lo ha hecho justamente al denunciar el carácter espurio de sus respectivas deformaciones. Sin embargo, víctima de un destino de incomprensión, su obra sufre una lectura paradójicamente injusta. En efecto, cuando defendió la magia natural, en cuanto culminación de las ciencias de la Naturaleza, fue tomado por hereje - o, cuanto menos, por heterodoxo por parte de sus mismos contemporáneos; en cambio, cuando ahora, en las Disputationes, combate las supercherías, la posteridad, en la figura de algunos de sus intérpretes, comete el error de creer que está desmintiendo esa primera defensa.

En este texto, Pico ataca, pues, la supuesta injerencia de influencias espirituales sobre el mundo natural y humano. Y no lo hace solamente porque ellas cuestionarían el libre albedrío del hombre, es decir, por el determinismo que la posición astrológica implica, sino simplemente porque, al igual que la goeteia, la juzga falsa.

\section{IV}

De hecho, el pensamiento agustiniano había discurrido por vías similares. Al comentar el pasaje bíblico sobre las estrellas "que fueron puestas en las constelaciones y en las épocas", el Hiponense aclara que esta referencia no es al supuesto determinismo astral, sino a las nociones astronómicas necesarias para la vida y estudiadas por los navegantes y agricultores. Y recuérdese que algo muy similar se encontraba respecto de lo oculto de la magia, a propósito del ejemplo agustiniano de las piedras magnéticas. Ciertamente, no es casual que Pico lo cite, ${ }^{19}$ ya que llama en su apoyo a la autoridad de Agustín para insistir en una distinción que no consideraba debidamente nítida en su época y, por ende, peligrosa para espíritus poco instruidos.

Disputationes adversus astrologiam divinatricem, I-IV, a cura di E. Garin, Firenze, Vallecchi, p. 41.

Cfr. Lubac, H. de, Pic de la Mirandole. Études et discussions, Paris, Aubier Montaigne, 1974, pp. 317-318. Respecto de la alineación mencionada de astrología-magia, tanto en sentido recto como en el perverso, Henri de Lubac afirma con acierto: "[...] maintenant les 'Disputationes' raillent les 'délires' d'une magie qui provient de la même erreur que cette astrologie divinatrice [...]". 
Cabe sintetizar ahora las convergencias y diferencias que se pueden trazar en las posiciones de Agustín y Pico sobre la magia. Por nuestra parte, diríamos que, en general, las primeras se dan en lo que hace al contenido doctrinal de fondo en ambas, mientras que las divergencias obedecen fundamentalmente a sus respectivos contextos históricos y a la terminología.

En efecto, en lo que concierne a las convergencias:

1) ambos asignan a lo oculto y a lo que produce estupor dos valores opuestos: el positivo, en cuyo caso se trata de potencias naturales descubiertas y actuadas por el hombre; y el negativo, en cuyo origen presentan, sin embargo, matices de diferencia.

2) los dos sostienen esa distinción tanto en lo que hace a la astrología como a la magia.

3) en lo que toca a lo oculto en sentido positivo, ambos lo asumen especialmente en el plano objetivo, que llamamos "extrínseco y universal", lo que, en el caso de Pico se funda en la noción de sympatheia, y en el agustiniano, en la de Ordo universalis.

En cuanto a las divergencias, se pueden señalar las siguientes:

1) con el fin de evitar equívocos, Agustín toma la palabra "magia" sólo en sentido negativo, acercándola como término a goeteia, en lugar de oponerla a éste. Pico, aunque sin éxito, intenta subrayar la distinción. Esto responde a las distintas preocupaciones fundamentales de uno y otro y, por así decir, a los públicos a los que se dirigen: la agustiniana es una misión fundamentalmente pastoral; la de Pico es concebida en el círculo de los intelectuales del Quattrocento.

2) respecto del origen de la goeteia, Agustín insiste, mucho más que el Mirandolano, en la intervención demoníaca; Pico, en cambio, parece imputarla en mayor medida a intereses humanos, tanto de poder como de riqueza. El primero tiende a proyectar la procedencia del mal en un plano extrínseco, trascendente al hombre; el segundo, precisamente por su carácter de humanista, hace girar todo en torno de la condición humana.

3) el punto más importante en las diferencias entre la posición de Agustín y de Pico sobre el tema de la magia y de lo oculto es, sin duda, el que concieme a su último sentido positivo. Y aquí se da algo semejante a lo que advertíamos en el punto anterior. La actitud agustiniana ante lo oculto de la naturaleza es la de invitar a la adoración, a abismarse en la contemplación de las maravillas creadas por Dios. La del humanista, en cambio, sobre esa misma base, incita a las operaciones humanas.

En efecto, recordemos que, según la concepción piquiana, la magia es la parte práctica de las ciencias de la Naturaleza, y su culminación. Precisamente porque saben penetrar en las razones ocultas de lo natural, las artes mágicas no sólo admiten la intervención humana responsable, experta y eficaz, sino que culminan en ella. Desde esta perspectiva, no hay rectificación alguna en las Disputationes adversus astrologiam divinatricem respecto de los textos anteriores del Mirandolano en relación con este tema, ya que su polémica tiene como blanco principal el supuesto determinismo astrológico que atentaba contra la libertad. En 
la misma linea, F. Yates ha visto claramente que "las operaciones de las fuerzas cósmicas en la magia renacentista, que no es lo mismo que la medieval, prefiguran el gran movimiento científico de los tiempos-por venir. El universo animado, sobre el que se opera a través de la magia, cambia eventualmente en el universo matemático, sobre el que operará siglos después la mecánica". ${ }^{20}$

Contemplación gozosa de los secretos de la Naturaleza creada por Dios en Agustín, invitación al obrar humano en Pico, la reivindicación de la magia natural en este último no es otra cosa que la reivindicación del poder que tiene el hombre de controlar su destino a través de la ciencia.

20 Cfr. Yates, F., op. cit. (nota 9) p. 197. 